\title{
Biosensing cytokine IL-6: A comparative analysis of natural and synthetic receptors
}

\author{
Eleonora Alfinito ${ }^{1,}$, , Matteo Beccaria ${ }^{2}$, and Mariangela Ciccarese, $\mathrm{MD}^{3}$ \\ 1 Department for Innovation Engineering, University of Salento; eleonora.alfinito@unisalento.it \\ 2 Department of Mathematics and Physics "Ennio de Giorgi”, University of Salento; \\ matteo.beccaria@unisalento.it \\ 3 Oncology Unit "Vito Fazzi" Hospital-Lecce; mari.ciccarese75@gmail.com \\ * Correspondence: eleonora.alfinito@unisalento.it
}

\begin{abstract}
Cytokines are a family of proteins which play a major role in the regulation of immune system, and enter the development of several diseases from rheumatoid arthritis to cancer and, more recently, COVID-19. Therefore, many efforts are currently being spent in therapy and diagnosis, producing inhibitory drugs, as well as biosensors for a rapid, poor invasive, and effective detection. In this regards, even more efficient cytokine receptors are under investigation. In this paper we analyze a set of receptors of cytokine IL-6, investigating their topological features by means of a theoretical approach. Our results suggest a topological indicator that may help in the identification of those receptors having the highest complementarity with the protein, a feature expected to ensure a stable binding. Furthermore, we propose and discuss the use of these receptors in an ideal experiment.
\end{abstract}

Keywords: IL-6 cytokine; biosensors; Proteotronics; aptamers; topological analysis.

\section{Introduction}

In the recent COVID-19 pandemic, anti-inflammation therapies were recommended to reduce medical complications. As a matter of fact, subgroups of patients with severe form of COVID-19 have developed a hyperinflammation syndrome [1]. In particular, high levels of cytokine IL-6 pushed the clinical trials of specific inhibitors of this protein and of Janus kinase (JAK) on COVID-19 patients in China [1,2]. The improvement of patient health and the modest side effects suggest to use the IL-6 antagonist therapy not only against this virus but also in the treatment of other lethal viruses [2].

On the other side, the role of cytokines in several diseases, from rheumatoid arthritis to cancer, is well known [3-5]: After IL-6 is synthesized in a local lesion in the initial stage of inflammation, it moves to the liver through the bloodstream, followed by the rapid induction of an extensive range of acute phase proteins such as C-reactive protein (CRP), serum amyloid A(SAA), fibrinogen, haptoglobin, and a1-antichymotrypsin [6]. In view of the range of biological activities of IL- 6 and its pathological role in various diseases, IL-6 targeting have constituted a novel treatment strategy for various immune-mediated diseases. The development of tocilizumab was a direct result of this hypothesis. Tocilizumab is a humanized anti-IL-6 R monoclonal Ab of the IgG1 class that was generated by grafting the complementarity determining regions of a mouse antihuman IL- $6 \mathrm{R} A \mathrm{~b}$ on to human IgG1 [7], and it blocks IL-6-mediated signal transduction by inhibiting IL-6 binding to transmembrane and soluble IL-6R. Recently, tocilizumab represents an area of investigation in clinical care for the treatment of severe COVID-19 pneumonia [8-10]. 
The relation of inflammation and various types of cancer has been suggested by several studies. Recently, it has been showed that anti-inflammatory agents can attenuate tumor growth in the breast cancer-bearing mice [11], implying the importance of inflammatory microenvironment in tumor growth. Malignant cells exhibit a high proliferation, which can be enhanced by inflammation. The inflammatory molecules in the tumor microenvironment are mainly secreted by tumor cells themselves and/or other stromal cells [12].IL-6 plays a critical role in the expansion and differentiation of tumor cells $[13,14]$, and can also modulate a tumor therapeutic resistance such as multidrug resistance (MDR) [15], whose engagement triggers activation of JAK, and the downstream effectors STAT3, SHP-2/ Ras, and PI3K/Akt [16]. It has been showed that IL-6 levels are significantly elevated in lung and breast cancer patients associated with poor prognosis; moreover, IL-6 can affect all aspects of tumorigenesis process by regulating proliferation, apoptosis, metabolism, survival, angiogenesis, and metastasis [17]. Blocking IL-6 and/or its receptor could be a trigger point to cure tumors associated with high levels of this cytokine, such as breast cancer.

Several drugs have been produced to contrast IL-6 activity [18], and the detection of very small alterations of cytokine levels [19], and specifically of IL-6, could be helpful in the early recognition of an inflammatory state [20-24]. Biosensors may use aptamers, antibodies, and also antibody fragments as detection elements [25]. Their performances, pros and cons, have been recently discussed in [25]: Aptamers usually show very high affinity to the ligand and have a very small size, which allows to produce a high density sensing element, thus reducing the LOD (limit of detection) of the detector. On the other hand, Fabs have the benefit of cost effective processes and fast production.

IL-6 cytokine belongs to the family of gp130-coupled cytokines, i.e. proteins that transfer the signal through this glycoprotein, also known as the $\beta$-receptor. In the process of activation, IL-6 firstly forms a dimer with the $\alpha$-receptor IL-6R, then a hetero-trimer with gp130. It has been postulated that only in the hexameric quaternary structure IL-6 is able to properly work [26,27]. Several artificial receptors have been tested against IL-6, IL-6R, and gp-130 [18], some of those entered and passed the clinical trials [7-10,18], while others remain in the labs [28,29]. All these receptors show high affinity to the ligand and specific inhibitory abilities. Both affinity and inhibition are the results of many factors, one of these is a good topological complementarity. In the typical scenario, IL-6 and its receptors undergo a conformational change when they bind together, and the cytokine seems to be able to assume different forms, adapting its shape to the receptor [18]. For example, a massive conformational change has been claimed at the origin of the inhibition mechanism of IL-6 due to the clinical Fab olokizumab (OKZ). Indeed, its binding induces some amino-acids of the cytokine, usually assembled as a random coil, to conform like a small helix that occludes the gp130 binding site (Site 3), and inhibits the hexamer formation. In this case inhibition seems to have a peculiarly steric origin.

Although the concept of affinity is qualitatively simple to understand, it is not uniquely defined at the quantitative level. Often it is given in terms of the dissociation constant $\mathrm{K}_{\mathrm{D}}$, defined as the ratio of two dynamic constants $k_{\text {off }} / \mathrm{k}_{\mathrm{on}}$, the off/on rate constants, which are themselves used to measure affinity. Specifically, $K_{D}$ describes the dynamic equilibrium of two species which combine in a product (the complex) and of the product which decays in the two species. As small as $K_{D}$ is, as high is the affinity. On the other side, the role of koff [30,31], which signal the life-time of the complex, is becoming even more relevant, in particular in pharmaceutics, due to the performance discrepancy between drugs tested in vitro/silico or in vivo [30].

Finally, besides clinical uses, we are interested in understanding whether the best-fitting arrangement of the receptor-ligand pair can be quantified. To this purpose, IL-6 offers an uncommonly wide set of different choices (receptors), between aptamers [32,20,21] and Fabs [22,23]. Aptamers are relatively young synthetic biomolecules [33] made of single strands of DNA or RNA selected in vitro to bind with high affinity a specific ligand (proteins, viruses, ions). Their outstanding performances, in vitro and also in vivo, in terms of affinity and stability, give them an even more important role in therapy and diagnosis [34,35]. Furthermore, the interest in 
pharmaceutics [36] has pushed forward also the investigation of their 3D structure with crystallographic analysis [32,37,38] and also computational analysis [39-41].

Fabs are antibody fragments, produced in labs with cloning techniques and have also good affinity with the ligand. In general, they are less customizable than aptamers and are more prone to denaturation, although, at present, they remain the best choice for who needs cheap and also quickly available material [25].

To shed light on the mechanisms of binding, the 3D structures of some of the IL-6 receptor complexes have been resolved using a crystallographic analysis [38]. Remarkable differences in the way receptors bind the ligand have been highlighted. The aim of the present investigation is to compare these receptors on a totally topological basis, in order to identify their features, performances, and limits in the perspective of their utilization in sensing devices. The proposed method takes advantage of public and freely available data [38] and of a theoretical approach known as Proteotronics [42]. At present, there are several different entries of IL-6 alone, i.e. in its native state, or complexed with different receptors. The list of the analyzed structures is in Table 1, and, besides the natural IL-6.IL-6R-gp-130 assembly, it contains the cytokine complexed with some Fabs and a Slow-Off-Rate (low dissociation rate with the ligand) Modified aptamer (SOMAmer) [32]; this novel kind of aptamer has been designed to implement the affinity to ligands, specifically to those usually quite difficult to bind [43].

The choice of the most appropriate receptor for a biosensor is often biased by the experience that researchers have with it. This may happen for economical reasons and also because the expected performances of different receptors are difficult to compare. Here we outline a procedure to attempt such a comparison, at least within a specific theoretical approach, by analyzing the topological features of different receptors complexed with IL-6.

To this aim, we use a theoretical approach [42] which translates the main topological elements of the assigned biomolecule (a protein, an oligonucleotide, a complex) into an interaction network. This complex network is made of nodes and links, one node for one nucleotide/amino-acid, one link for a pair of nearest neighbor nodes.

Once the biomolecule is mapped onto the associated network, it can be explored allowing a flow of electrical current to permeate it. This kind of theoretical probing is able to detect the internal structure of the network and, in particular, to reveal the presence of short-paths or bottlenecks [44]. In the present case, the spreading of the current flow aims to detect the quality of the binding. We have compared the receptors listed in Table 1 and introduced an indicator that is able to sort the topological complementarity with the ligand (IL-6). As expected, the clinical Fab is one of the best performing options, although another Fab as well as the SOMAmer are competitive with it. Finally, we propose predictions about the expected performances of these receptors when used in an ideal sensor.

\section{Materials and Methods}

\subsection{Materials}

All data concerning the present investigation is available in the public data bank repository, hereafter called PDB [38]. In the following we give a sketch of each of them:

- $\quad$ IL-6

The 3D structure of this protein has been resolved in the native state, PDB ID: 1alu [45], i.e. the protein alone in the minimum of conformational energy, and also complexed with several receptors $[18,26,28,29,32]$. It is made of 4 main helices, sequentially named A,B,C,D, and long about 20 amino acids, and a $5^{\text {th }}$ small helix, say E, of 12 residues. To the aim of present analysis we will refer to the active state as the state the protein assumes when complexed with a receptor. In general, active and native state refer to different protein conformations, and, specifically, in IL-6, the shape of the active state depends on the receptor.

- $\quad$ IL-6R 
The $\alpha$-receptor of IL-6 (IL-6R) is a 3 domain protein (D1, D2, D3) and has been expressed and resolved completely in [46], in the absence of any ligand (native state), and also complexed with IL-6R and gp130 [26]. IL-6R is itself relevant for disease detection since increased values can be found in patients suffering of different inflammations illnesses [46]. Only two domains, say D2, and D3 are reported in [26] and they will be used to compare native and active state. If differences between these conformations are due to D1, they cannot be detected.

- $\quad$ SOMAmer SL1025

SOMAmer (Slow Off-rate Modified Aptamer) is a new kind of DNA aptamer obtained by introducing modified moieties in the standard nucleotide catalog. This characteristic is of high interest in producing effective drugs, because it avoids metabolic degradation and a rapid clearance [47]. SOMAmers are effective against many ligands and also proteins usually considered challenging $[43,48]$. In particular, SOMAmer SL1025 [32,48] binds with high affinity $\left(K_{D}=0.2 \mathrm{nM}\right)$ human IL-6 protein and blocks the binding with IL-6R [49]. It is efficacious against rheumatoid arthritis, at least in animal trials [43,49]. SL1025 3D structure has been deposited in two different entries, named 4ni7 and 4ni9 [32]. They are very similar and the latter is the most complete, therefore, we limit our study to 4ni9 (chain A for the cytokine and B for the aptamer). The aptamer induce a poor or null conformational change in the protein, although it is presumable it strongly adapts its native shape to obtain a large complementarity with protein.

- Olokizumab (OKZ)-Fab portion

Olokizumab is a humanized monoclonal antibody, whose Fab fragment has been resolved and is available in PDB at the entry 4cni [18]. It has very high affinity to the cytokine IL-6, say, $\mathrm{K}_{\mathrm{D}}=10 \mathrm{pM}$. This so high affinity is due, at least in part, to the high level of interconnection between the two biomolecules. The presence of OKZ induces a particular conformational change in IL-6. Crystallographic analysis revealed a noticeable difference with respect the natural assembly IL-6R-IL-6.gp130, i.e. a set of few amino-acids from GLU42-ASN47, detected in other complexes as a random coil, here appears organized in a small helix, named from here after, helix F. This small helix occludes the gp130 receptor-binding pocket, thus producing the high inhibitory activity of this antibody. At present OKZ is in the Phase III trial on patients with rheumatoid arthritis [50].

- $\quad 61 \mathrm{H} 7$ \& 68F2- humanized Camelid-Fab

61H7 and 68F2 are neutralizing IL-6 antibody fragment. Antibodies were obtained upon Llama immunization with human IL- 6 and exhibit ultra-high affinity to the cytokine, specifically, $\mathrm{K}_{\mathrm{D}}=$ 3.3-6.3 pM (61H7) , and $\mathrm{K}_{\mathrm{D}}=13-21 \mathrm{pM}$ (68F2) [29] . On the other hand, the way they bind IL-6 is quite different: both were selected to block Site I of the cytokine, i.e. mimicking the action of IL-6R, but while $61 \mathrm{H} 7$ binds the top of the IL-6 helix bundle, $68 \mathrm{~F} 2$ is set transversally toward the same side (see Figure A2).

Table 1. List of PDB [38] entries used in present paper. Entries 1alu and 1n26 refer to the native states of IL-6 and IL-6R, respectively.

\begin{tabular}{ccc}
\hline \#entry & Ligand & Receptor \\
1p9m & IL-6 & gp130, IL-6R \\
1alu & IL-6 & n.a. \\
1n26 & n.a. & IL-6R \\
4 zs7 & IL-6 & Fab 68F2 \\
$4 \mathrm{cni}$ & IL-6 & Fab OKZ \\
4 o9h & IL-6 & Fab 61H7 \\
4ni9 & IL-6 & SOMAmer SL1025 \\
\hline
\end{tabular}

\subsection{Methods}

We used an investigation technique named Proteotronics [36, 40-42,44,51] which explores the electronic responses of biomolecules by using a complex network approach. The technique is intuitive and simple to use. The main idea is to map the biomolecule tertiary structure (3D) into a 
graph, which accounts for both its structural and physico-chemical properties. Each node corresponds to a nucleotide/amino-acid and contains all information we need to account for the interaction we have to describe (barycenter position, resistivity, temperature, dielectric constant, elastic module, and so on). Undirected links may connect two nodes. This happens depending on the entries of the distance matrix describing the graph. It is a zero-diagonal symmetric matrix with dimension given by the number of nodes and $(i, j)$-th entry equal to the distance between the $i$-th and $j$-th nodes. In this modelling, the presence of an activated link represents the existence of an interaction between the nodes. Because each known interaction is distance-sensitive, a link is drawn only if the nodes are closer than as assigned cut-off distance, $D$. In such a way, we can figure the nodes like soft spheres of radius $D / 2$ that interact only when they overlap. The connected nodes are the nearest neighbor nodes, the only ones able to interact. Increasing the parameter $D$, the number of links increases. The distance matrix is therefore mapped into an adjacency matrix, $A$, which stores zero when the distance between the $i, j$ nodes, $l_{i j}$, is larger than $D$, and else stores 1 . The graphical representation of $A$ is called a contact map (CoMa) and gives a prompt sketch of nearest neighbor nodes and, definitely, describes the skeleton of the biomolecule.

In the present analysis, we are interested in electronic interactions and therefore an elementary impedance is associated to each pair of connected nodes, as follows [42,51]:

$z_{i j}(\omega)=\frac{l_{i, j} \times \rho_{i, j}}{S_{i, j}\left(1+i \rho_{i, j} \varepsilon_{0} \varepsilon_{i, j} \omega\right)^{\prime}}, \quad S_{i j}(D)=\frac{\pi\left(D^{2}-l_{i, j}^{2}\right)}{4}$

where $\rho_{i, j}, \varepsilon_{i, j}$, and $S_{i, j}$ are the resistivity, the relative dielectric constant, and the intersection area of the pair of connected nodes, respectively. The resistivity and relative dielectric constant may be calculated as described in [51]. Notice that the electrical response, as defined in Equation (1), depends on the biomolecule 3D structure. Impedance reduces to the simple resistance when $\omega=0$. The resistance spectrum, $r(D)$ is calculated by allowing the value of $D$ to increase, and has a decreasing shape. Most frequently, in present investigation, we will use the relative resistance spectrum $\operatorname{rr}(D)$ i.e. the ratio of two resistances (see later) calculated for increasing values of $D$.

Our analysis starts with the investigation of the topology of the biomolecule equivalent network. We allow an electron flow to go inside the protein-receptor complex, thus detecting its internal structure like shortcuts and bottlenecks. To this aim, in our analysis we do not account for the specific electronic properties of each node. Instead, we assume the same value of resistivity for all (here $\rho=10^{14} \Omega \AA$ ). Specifically, we imagine IL-6 (hereafter, the ligand) as the source and the sink of an electrical current that flows through the receptor when it is sufficiently close to the ligand. A pair of ideal contacts connects the ligand to an ideal battery working in D.C. in the linear regime. The global response is well described by that of two resistances in parallel, one associated to the ligand, the other to the receptor [40]. As a consequence, the resistance of the complex is always smaller than that of the ligand. When the cut-off distance $D$ is too small (here below about $4 \AA$ for Fabs and $8 \AA$ for the SOMAmer) no interlink can be drawn, because the parts are too far. In this case, the flow goes only inside the ligand and we have $r r \cong 1$, with $r r$ being the ratio of the complex resistance to the receptor resistance. At increasing $D$, the number of outside links (links between receptor and ligand) grows and some amount of current flows through them, leading to an even smaller complex resistance. Finally, for sufficiently large value of the parameter $D$, the ratio $r r$ saturates as soon as the network becomes completely connected.

\section{Results}

In this section we first describe the topological structures in terms of contact maps. We then introduce a new (topological) indicator that may be used to characterize the quality of binding. Finally, we propose an ideal experimental setup (i.e. a Gedanken-Experiment). 


\subsection{Contact maps}

As we already mentioned, in our approach the interaction network is represented by its contact map (CoMa). A CoMa is a picture of the nearest neighbor nodes for an assigned value of $D$. Each point of coordinates $(i, j)$ is drawn only if the corresponding nodes $i$ and $j$ are connected. The number of connected nodes grows with $D$, reaching the maximum value, $\mathrm{M}(\mathrm{M}-1) / 2$, ( $\mathrm{M}$ is the number of nodes) for high values of $D[42,44]$. Furthermore, the plot of connected nodes is specific for each biomolecule and helps to identify its secondary structure (Figure 1a). Protein IL-6 adapts its shape to the receptor, as is revealed comparing the CoMas of its native and active state. Each CoMa corresponds to the network adjacency matrix, which is symmetric. Therefore, it can be drawn completely (full picture), or only considering $i<j$ (or $j<i$ ) (half picture): in this latter case, the other half of the picture is used to draw another adjacency matrix. For example, in Figure 1a we report the IL-6 CoMa (full picture) in its native state (PDB ID: 1alu [45]), while in Figure $1 \mathrm{~b}$ the half map of the native state is compared with the half map of IL-6 in the active state produced by the conjugation with its natural receptors, IL-6R and gp130 (PDB ID: 1p9m [26]). This choice has been also adopted for Figure 2, where the active states of IL-6, due to the conjugation of SOMAmer SL1025 (PDB ID: 4ni9[32]), OKZ (PDB ID: 4cni [18]) and two humanized Camelid Fabs (PDB ID: 4o9h [28]), and (PDB ID: 4zs7 [29]) have been compared with the IL-6 native state. This comparison suggests modest differences between the native and active state of IL- 6 bound to its natural receptors or with SOMAmer.

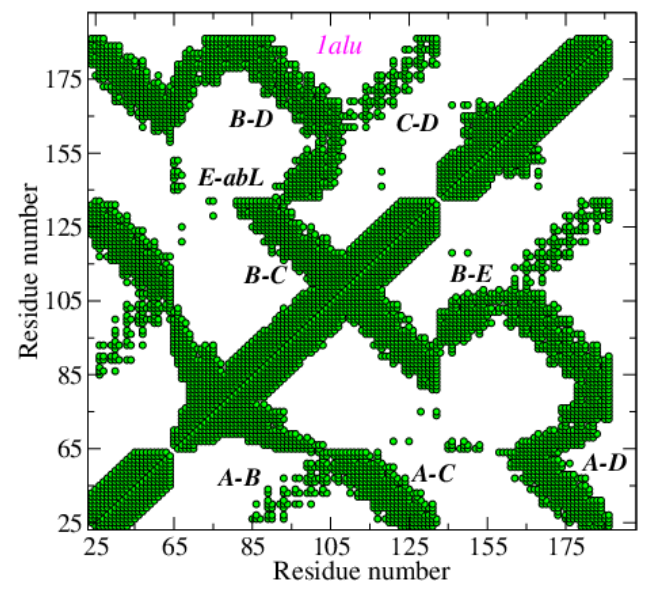

(a)

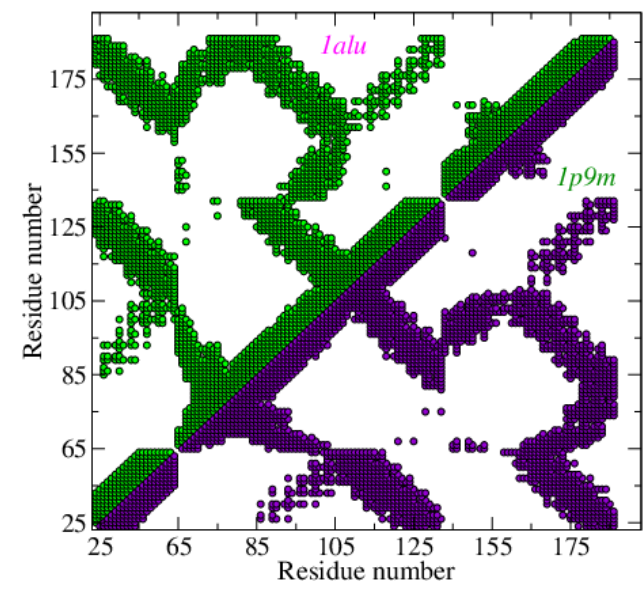

(b)

Figure 1. Contact maps of IL-6, in: (a) the native state (PDB ID: 1alu [45]) and (b) the active state due to the conjugation with natural receptors IL-6R and gp130 (PDB ID:1p9m [26]), D=15 ̊. (a) The intra-contacts among helices $\mathrm{A}, \mathrm{B}, \mathrm{C}, \mathrm{D}, \mathrm{E}$ are highlighted, $a b L$ indicates the loop between helices $\mathrm{A}, \mathrm{B}$.;

(b) IL-6 in the native state (green) is reported vs IL-6 in the active state (violet).

On the other side, Fabs are found to induce a more relevant conformational change in the protein, mainly due to helix E. This helix shifts towards the AB loop and away from helix C. To the aim of a faithful comparison, the same strands of IL-6 have been used for all the entries, both in Figure 1 and Figure 2, in particular, a set of 133 of amino acids from GLU 10 to MET 171. Of course, differences due to protein moieties not present in this set, cannot be detected in this regard. It has been reported that, when complexed with OKZ, IL-6 shows a small helix (F), not resolved in other experiments, thus away from the previous selection; this helix is probably the responsible of the occlusion of Site III, and this, in turn, inhibits signal transmission [18]. Information about the role of this helix can be obtained by looking at the CoMa of the complete complex (Figure 4b). 


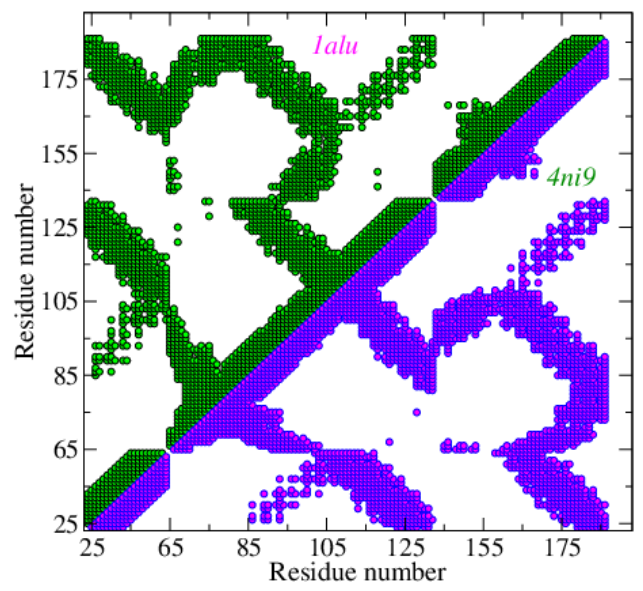

(a)

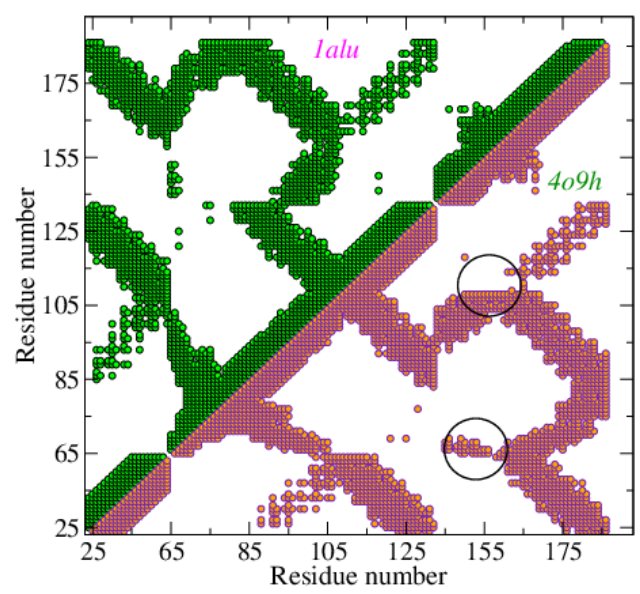

(c)

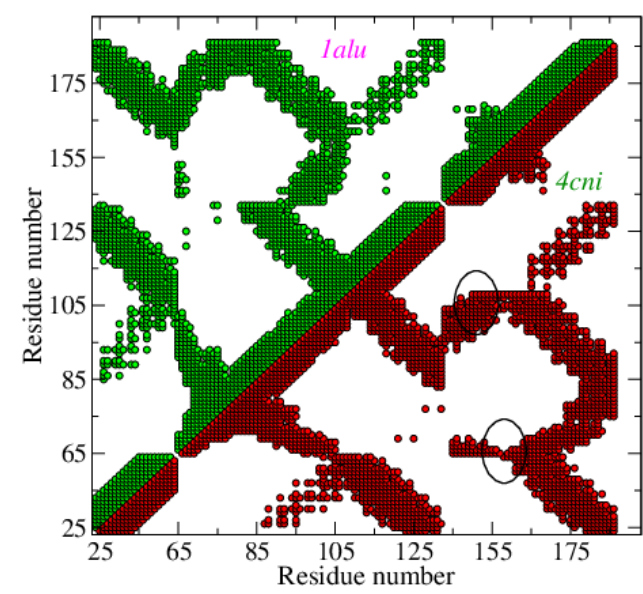

(b)

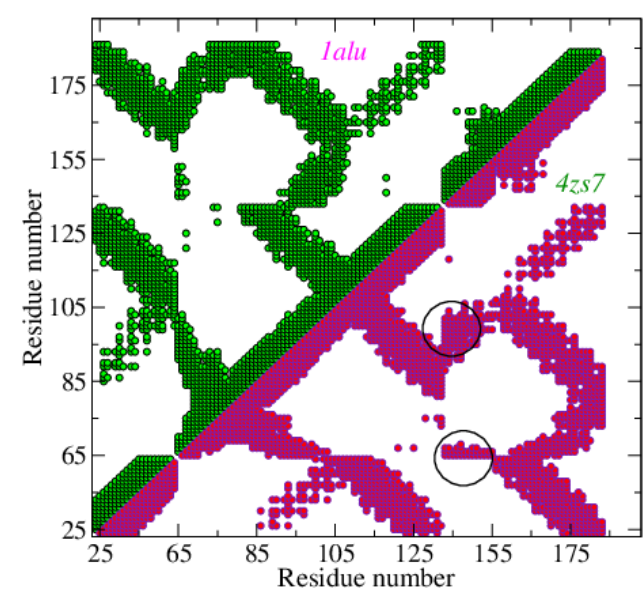

(d)

Figure 2. Contact maps of IL-6, in the native state and the active state due to the conjugation with different receptors, $D=15 \AA$. (a) IL-6 in the native state (green) vs. IL- 6 in the active state due to conjugation with SL1025 SOMAmer (blue); (b) IL-6 in the native state (green) vs. IL-6 in the active state due to conjugation with OKZ Fab(red); (c) IL-6 in the native state (green) vs. IL-6 in the active state due to conjugation with $61 \mathrm{H} 7 \mathrm{Fab}$ (pink); (d) IL-6 in the native state (green) vs. IL-6 in the active state due to conjugation with 68F2 Fab (magenta). Main differences with the native states have been marked with an ellipse.

When the complexes are analyzed, the differences among them appear much more relevant. Using as a benchmark the natural trimer, its CoMa, taken at $D=15 \AA$, shows a high number of interlinks with both receptors. In particular, IL-6R ( $\alpha$-receptor) has a preferential binding with helices A,B, and D, while gp130( $\beta$-receptor) appears more close to helices A and C (see Figure 3). The SOMAmer elicits a very similar kind of binding and, despite its small size, spans a very large number of links with IL-6 (mainly with helices A,C, and D) in a very good mimicking of the natural receptors (see Figure 4a). 


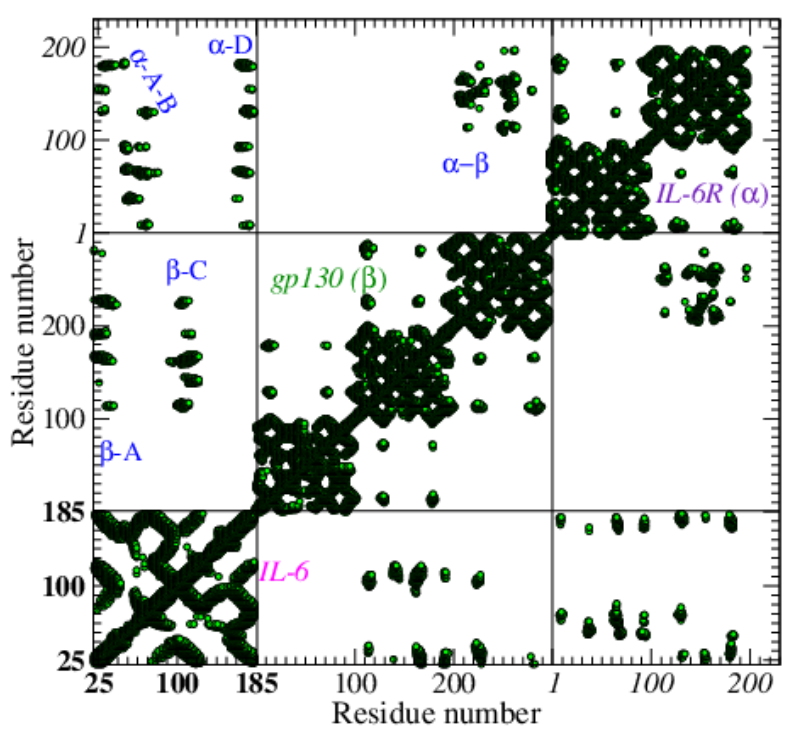

Figure 3. Contact map of IL-6 complexed with its natural receptors, IL-6R, the $\alpha$-receptor, and gp130, the $\beta$-receptor, PDB ID: $1 \mathrm{p} 9 \mathrm{~m}$ [26], $D=15 \AA$. The three boxes on the diagonal contains the intra-contacts of IL-6, gp130, and IL-6R, respectively. Outside contacts (between protein and receptors) are in the lateral boxes. Some of the best resolved receptor $(\alpha / \beta)$-protein contacts are highlighted. Progressive node number are indicated for IL-6 (in bold), gp130, and IL-6R (in italic).

Both chains $\mathrm{H}$ and L contribute to the binding, in all Fabs. All Fabs bind helix D, the two Camelid Fabs also bind helix A; small helices E, F have a main role in the complex with OKZ. Fab 61H7 has a huge number of links with helices B and C.

\section{1. $\mathrm{ToCI}$}

The topological complementarity index $(T o C I)$ is a measure of the complementarity of the parts of the complex. It accounts for the closeness of the shared surfaces and their extension. It is defined as the normalized integral of the relative resistance spectrum $r r \equiv r r(D)$, where here $r r$ is the ratio of the resistance of the IL-6.receptor complex to the resistance of IL-6. Specifically, the electrical flow is sent inside IL-6 and from this to the selected receptor.

The $r r$ spectrum contains useful information about the binding: at the smallest $D$ values the ratio $r r$ can be large as 1 , thus signaling a very few number of outside links (IL-6.receptor links), or may shows a small growth toward a maximum smaller than 1 . In the latter case, the rate of growth of outside links is similar to that of intralinks (inside IL-6), thus suggesting a complex made of two very close parts. In general, the smaller the value of the maximum, the higher the number of outside links. Enlarging $D$, the intralink rate of growth increases. The sharper the $r r$ decrease, the larger the rate at which new links are drawn. The rapidity of decrease gives also an indication of the surface complementarity, because a larger number of links implies a larger common surface. Increasing $D$, the current flows more deeply inside the receptor and the role of the longest paths in both receptor and protein become less and less important, since they have the largest resistance. A saturation is reached when the current flows inside both protein and receptor along the shortest paths. In this condition the closest nodes are more important than the most separate ones. 


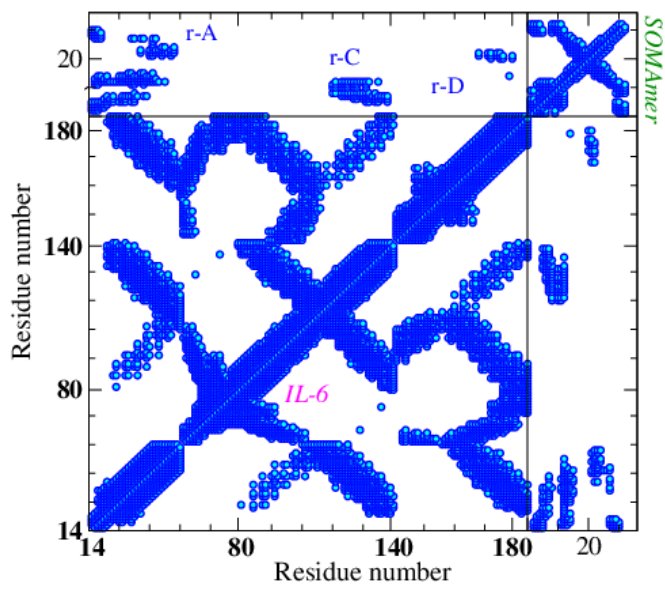

(a)

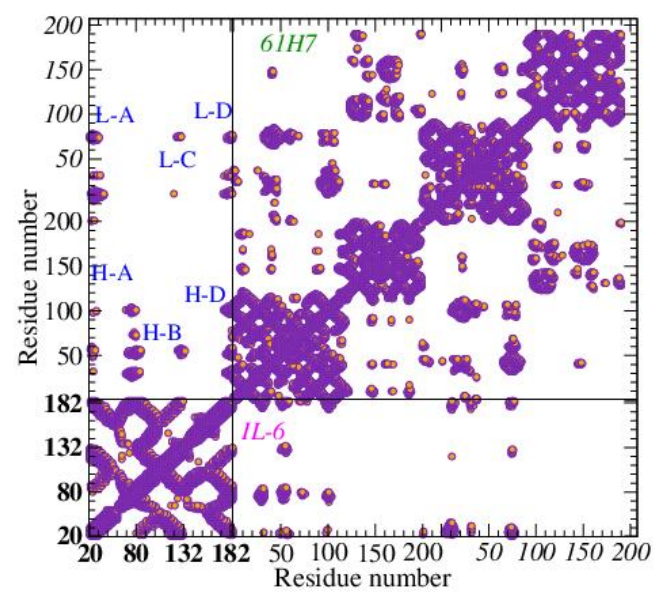

(c)

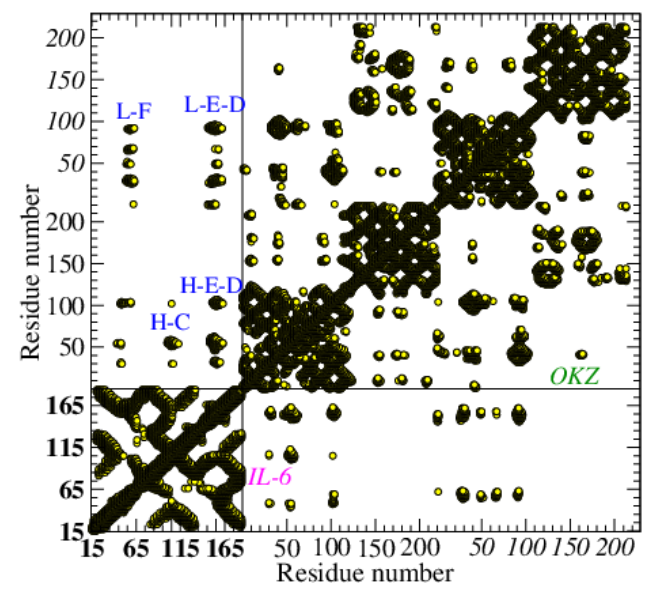

(b)

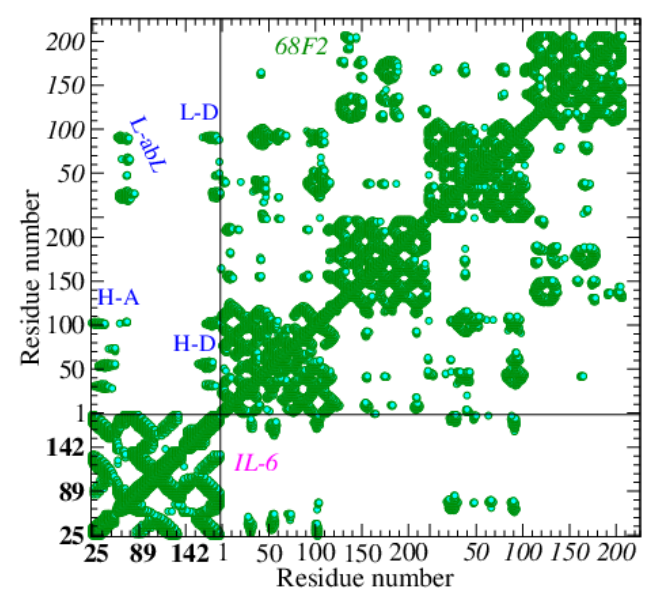

(d)

Figure 4: Contact maps of IL-6 complexed with different receptors, $D=15 \AA$. (a) SOMAmer SL1025: main links between the receptor ( $\mathrm{r}$ ) and helices A,C,D are indicated; (b) Fab OKZ: main links between 4 IL- 6 helices, C,D, E, F and the heavy $(\mathrm{H})$ and light $(\mathrm{L})$ chain of the receptor are indicated; (c) Fab 68F2 :main links between IL-6 helices A,D, and the heavy $(\mathrm{H})$ and light $(\mathrm{L})$ chain of the receptor are indicated; (d) Fab 61H7: main links between IL-6 helices A,D and the heavy (H) and light (L) chain of the receptor are indicated. Helices B and C show contacts with chains $\mathrm{H}$ and L, respectively. Progressive node number are indicated for IL-6 (bold), receptor heavy chain (normal), and receptor light chain (italic).

The calculation of $r r$ has been performed by using four different contact positions, to account for the different ways the receptors may bind the protein. In particular, Site I is the epitope to which IL-6R binds IL-6 and is roughly rendered by putting the contacts on helices A and D (GLU10-MET171) on the side of N terminal. Site III, where gp130 binds IL-6, is rendered putting the contacts on the same helices, on the opposite side (THR30-ASN142). Due to the primary role of helix $C$ in natural binding of IL-6, a contact pair is also put on helices A and C (THR30-ALA117), and, finally, as an intermediate position, both contacts are put on helix B (ASN66-SER95). The profile of $r r$ as $D$ is varied depends on the contact positions, showing in some cases very dramatic differences. The corresponding complexes are much more asymmetric than the others. This is the case of Camelid Fab 61H7 [28] and of OKZ [18]: 61H7 Fab binds IL-6 at Site I, therefore, the contacts on the 
first position give a very low value of the maximum of $r r$, a sharp decrease and a low asymptotic value (larger flux inside the receptor than in the ligand). On the reverse, when contacts are put in the second position, a larger value of the maximum, a smoother decreasing, and a higher asymptotic value (i.e. a smaller current in the receptor) are found. A specular result is obtained for OKZ, which targets Site III. Finally, neither contacts on helix B nor C improve the responses given by contacts put on helices A and D. Similar arguments explain the behavior of the other ligand-receptor complexes (see Figure 5).

To quantify these differences, we have introduced as an indicator a quantity that we name ToCI (topological complementarity index). Specifically, we calculate the area of $r r$ under the sensitive height and over the sensitive range of $D$, the equivalent surface (Figure 5). The sensitive height is the difference between the value of $r r$ for each $D$, and its asymptotic value, and the sensitive range of $D$ is the range from the minimum value of $D$ (here $8 \AA$ ) to the value corresponding to the $90 \%$ of the maximal sensitive height. Finally, to account for the different sizes of the complexes, the area is divided for the ratio of the complex/protein number of nodes. With such a definition, we aim to take into account both the maximum value of $r r$ and the rapidity of its decrease as well.

The value of ToCI is smaller when the contact position mimics that of the binding site. Specifically, three of the 4 non primeval receptors have very similar performances, namely Fab 61H7 (1.2) and SOMAmer SL1025 (1.6), when bound to Site I, and OKZ (1.9) when bound to Site III (see Table 2). In some cases, as $61 \mathrm{H} 7$ and $\mathrm{OKZ}$, the differences between data obtained putting the contacts on the correct position or the opposite, are very large. Finally, these results agree with the known very high affinity to IL-6 (see Section 2.1). The very good topological complementarity between these 3 receptors and IL-6 suggests that both SOMAmer SL1025 and Fab 61H7 could perform (at least when used to detect IL-6) not worse than OKZ.

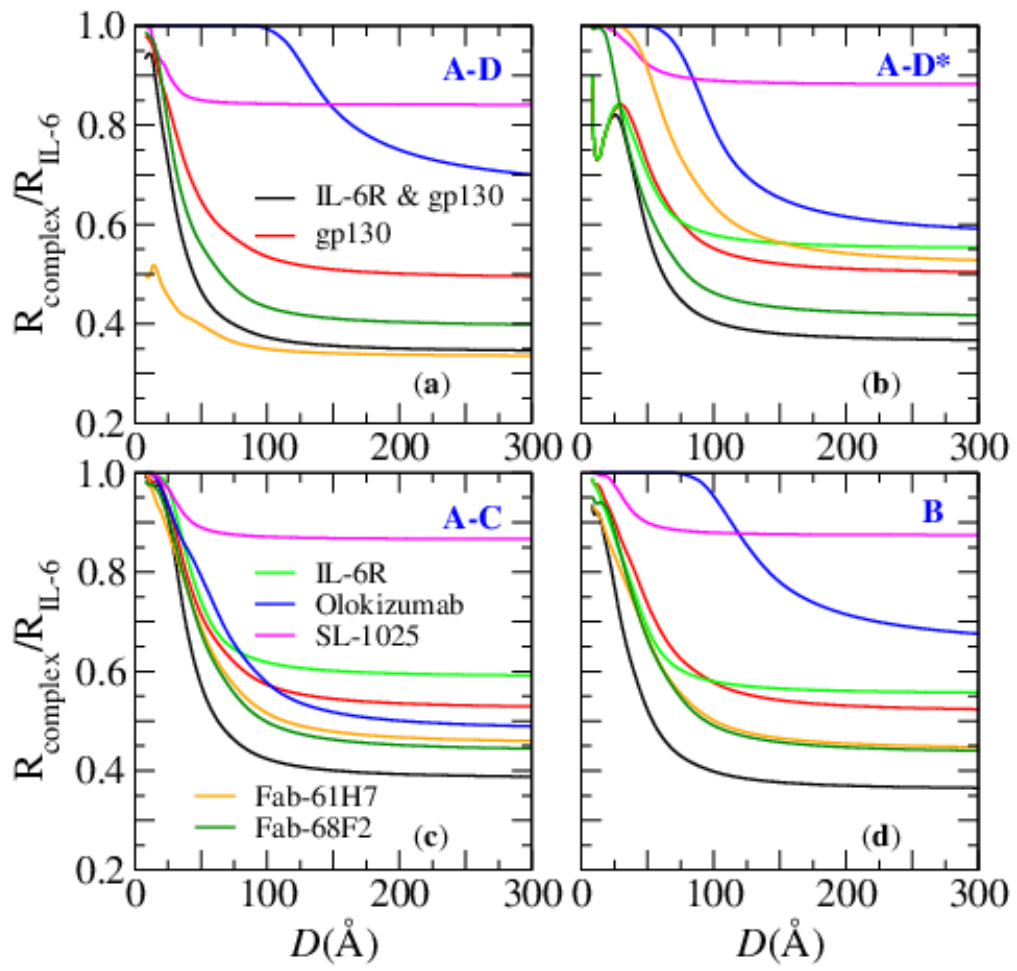

Figure 5. Relative resistance of IL-6 complexed with several receptors. Ideal electrical contacts are: (a) on helices A-D close to N-terminus, input/output GLU10/MET171; (b) on helices A-D in the opposite orientation (A-D*), input/output THR30/ASN142; (c) on helices A-C, input/output THR30/ALA117; (d) on helix B, input/output ASN66/SER95. 
Table 2. Table of topological complementarity index, ToCI, using 4 different contact positions. Lowercase s (\#) denotes the ToCI value, calculated putting the input/output contacts on IL-6 helices

(\#). Data refer to $r r$ spectra reported in Figure 5.

\begin{tabular}{lcccc}
\hline Receptor & s(A-D) & s(A-D $\left.{ }^{*}\right)$ & s(A-C) & s(B) \\
\hline IL-6R \& gp130 & $\mathbf{2 . 7}$ & 3.4 & 3.3 & 3.0 \\
gp130 & $\mathbf{4 . 1}$ & 4.4 & 4.9 & 5.1 \\
IL-6R & 3.1 & $\mathbf{3 . 0}$ & 5.2 & 3.5 \\
OKZ-Fab & 7.4 & $\mathbf{1 . 9}$ & 5.3 & 5.7 \\
SL1025-Apt & $\mathbf{1 . 6}$ & 3.0 & 2.2 & 2.4 \\
61H7-Fab & $\mathbf{1 . 2}$ & 6.7 & 4.4 & 4.2 \\
68F2-Fab & $\mathbf{3 . 4}$ & 4.2 & 4.4 & 4.1 \\
\hline
\end{tabular}

\subsection{Gedanken-Experiment}

A real biosensor is a customized and complex device whose performances mainly depend on the sensing element, signal transduction process, and amplification. Therefore, apparently, the description of an ideal sensor may appear unfeasible. Nevertheless, some common features may still be identified and make such a description meaningful. As a general requirement, a low limit of detection (LOD) is desirable. This is expected to imply a high affinity to the analyte. High affinity is a necessary condition to have a good selectivity. All the analyzed receptors have high/very high affinity to IL- 6 and are thus potentially good candidates. Besides a low LOD value, we also desire a good sensitivity i.e. a wide dose-response range. This means that the device (and in particular the receptors) gives sensitively different responses in a wide set of analyte concentration. The way the sensor translates the capture action is specific to the sensor itself. Here again, we focus on electrical ( impedance or resistance ) responses. In electrochemical sensors, a relevant measurement is that of the impedance spectrum. In particular, the zero frequency impedance value is associated to the so-called charge transfer resistance, Rст. Different doses are associated with different values of Rст. In quite different devices, for example FET/AFM devices[31], the analyte may be detected by conductance measurements instead of resistance ones.

Here, we compare the resistance of a biosensing element made of the previously investigated receptors (61H7 and 68F2 Fabs, OKZ, IL-6R, and SL1025) and try to predict the response at increasing concentration of the analyte (the dose response). Of course, speculations strictly apply to an ideal biosensor, i.e. such that the receptors are perfectly oriented, the noise/signal ratio is negligible, the electrode is perfectly functionalized, and so on. Nevertheless, our arguments are expected to establish bounds on the receptor performances.

Let us now describe the behaviour of the complex as the parameter $D$ is varied. In our modeling, it will be ideally traded for the analyte concentration. The resistance of the biosensing element, $R_{\text {sample, }}$ is given in terms of the resistances of the single receptor-ligand complex, $r_{c o m p}$, and the resistance of the receptor, $r_{\text {rec, }}[53,54,51]$. Specifically, when the receptors of the sample receive the analyte, a fraction $f$ of them binds the ligand. The extreme case $f=0$ means that the sample resistance is totally due to the receptors, while $f=1$ means that the sample resistance is totally due to the complexes. Furthermore, the storm of analytes strongly affects both receptors and complexes, raising their conformational energy [55]. In other terms, bonds become weaker and, in general, biomolecules are less rigid [55]. In our model, a bond reduction means a reduction of the value of $D$. Therefore, the complete variation of $f$ covers the range $\left(D_{0}, D_{1}\right), D_{0}>D_{1}$, with $D_{0}$ representing the condition $f=0$ and $D_{1}$ representing the condition $f=1$. In other terms, each dose corresponds to a specific $D$ value $[53,54,51]$. The total sample resistance reads

$$
R_{\text {sample }}(D)=N\left[f \times r_{\text {comp }}(D)+(1-f) \times r_{\text {rec }}(D)\right]
$$


where $N$ is a numerosity factor, e.g. it is the number of receptors in an ideal linear arrangment; more generally, it is the effective number of receptors as seen by the electrical contacts. The fraction of complexed receptors also depends on $D[53,54,51]$.

The ideal LOD is given by a single capture process, $f=1 / N$, i.e. the activation of the smallest part of the biosensing element:

$R_{\text {sample }}\left(D_{\text {LOD }}\right)=r_{\text {comp }}\left(D_{L O D}\right)+(N-1) \times r_{\text {rec }}\left(D_{L O D}\right) \approx N \times r_{\text {rec }}\left(D_{\text {LOD }}\right)$

where $r_{\text {comp }}\left(D_{L O D}\right)$ is assumed smaller than $N x r_{\text {rec }}\left(D_{L O D}\right)$.

Finally, when the dose is high, all the receptors bind the ligand, and $(f=1)$ :

$$
R_{\text {sample }}\left(D_{1}\right)=N \times r_{\text {comp }}\left(D_{1}\right)
$$

Measurements of electrochemical impedance $[20,21,51]$ often detect a growth of $R_{\text {sample, }}$ due to the injection of the analyte. From Equations (3-4), this means, for example, that $r_{\text {rec }}\left(D_{L O D}\right)$ is smaller than $r_{c o m p}\left(D_{1}\right)$. On the other side, assuming $r_{r e c}\left(D_{L O D}\right)>r_{c o m p}\left(D_{L O D}\right)$, it also means:

$$
r_{\text {comp }}\left(D_{L O D}\right)<r_{\text {rec }}\left(D_{L O D}\right)<r_{\text {comp }}\left(D_{1}\right) \text {, }
$$

and $D_{L O D}>D_{1} \quad$ (see Equation 1), i.e., arising the dose value, $D$ shifts toward lower values, in agreement with previous speculations. Finally, the wide range of $R_{\text {sample }}$ variation ( the sensibility) is given in terms of the single receptor/complex resistance (Equations 3 and 4). The exact range of $D$ can be matched to experiments. For the purposes of this section, i.e. with the aim of comparing the sensibility of different receptors, we can arbitrarily choose it as the widest possible. Specifically, here we select the sensitive D range, introduced in section (3.1), and perform the calculation of the complex resistance by putting the contacts on the receptor in order to mimic the real case (see Figure A1). This range surely overcomes the range in which any possible real device can work and has to be considered a guideline to estimate the real behaviour of the considered receptors.

A final remark about Equation 5 : the constrain $r_{r e c}\left(D_{L O D}\right)>r_{c o m p}\left(D_{L O D}\right)$ is straightforward when $r_{r e c}$ is the resistance of the receptor in its active state (appropriately deformed to accomodate the protein). On the other side, $r_{\text {rec }}$ should be meant as the resistance of the receptor in its native state ( ligand free). Among the selected receptors, the native state is known only for IL-6R, and, in general, the 3D structure of a biomolecule is known both in its native and active state only in few cases. On the other hand, for large $D$ values, the resistances of the native and active states are very close, because both networks have the same node number and the statistical error we make in using one rather than the other is negligible. Therefore, for our purposes, we will equate $r_{\text {rec }}$ with the resistance of the receptor in its active state, and this justifies Equation (5) .

Finally, the device sensibility is estimated by the difference $(\Delta)$ and the ratio $(\kappa)$ of the microscopic resistances:

$$
\mathrm{k}=\frac{R_{\text {sample }}\left(D_{1}\right)}{R_{\text {sample }}\left(D_{\text {LOD }}\right)}=\frac{r_{\text {comp }}\left(D_{1}\right)}{r_{\text {rec }}\left(D_{L O D}\right)},
$$

and :

$$
\Delta / N=1 / N\left(R_{\text {sample }}\left(D_{1}\right)-R_{\text {sample }}\left(D_{\text {LOD }}\right)\right)=r_{\text {comp }}\left(D_{L O 1}\right)-r_{\text {rec }}\left(D_{\text {LOD }}\right) .
$$


We found that SOMAmer SL1025 and Fab 61H7 have very high resistances, and also a very high resistance increment, $\Delta$, (see Table 3). On the other side, SL1025 has the smallest relative increment, $\kappa$, and Fab $61 \mathrm{H} 7$ the highest. In conclusion, both are able to convert the ligand capture in a large electrical response, in particular, Fab $61 \mathrm{H} 7$ can be used to explore a wider range of analyte concentrations, while SL1025 is able to give a strong signal also at the earliest capture event.

Table 3. Resistance data for the for the set of analyzed complexes. The resistances of the complex and the receptor, calculated in the ideal conditions of maximum response, $r_{c o m p}\left(D_{1}\right)$ and limit of detection (LOD), $r_{\text {rec }}\left(D_{L O D}\right)$, normalized to the value of $\operatorname{rrec}\left(D_{L O D}\right)$ of IL-6R, are reported as well as the corresponding receptor increment $\Delta$, and ratio $\kappa$.

\begin{tabular}{ccccc}
\hline receptor & $\boldsymbol{r}_{\text {comp }}\left(\boldsymbol{D}_{\boldsymbol{1}}\right)$ & $\boldsymbol{r}_{\text {rec }}\left(\boldsymbol{D}_{\text {LOD }}\right)$ & $\boldsymbol{\kappa}$ & $\boldsymbol{\Delta} / \mathbf{N}$ \\
\hline IL-6R & 16.4 & 1 & 16.4 & 15.4 \\
OKZ-Fab & 88.0 & 0.32 & 275 & 86.1 \\
SL1025-Apt & 227 & 15.9 & 14.3 & 211 \\
61H7-Fab & 200 & 0.38 & 530 & 200 \\
68F2-Fab & 35.9 & 0.33 & 110 & 35.9 \\
\hline
\end{tabular}

\section{Discussion}

Several diseases are accompanied by inflammatory states and high levels of cytokines. It is the case, for example, of cancer, where both malignant cell proliferation and also therapeutic resistance is enhanced by inflammation. In breast cancer, for example, cancer-associated adipocytes trigger radio-resistance by secreting IL-6 [56]. Therefore, targeting IL-6/JAK/STAT3 pathway can be considered as an effective therapeutic approach for cancers associated with overexpression of IL-6, including breast cancer [57-60].

Most recently, also COVID-19 patients in severe conditions benefitted from these therapies [8-10]. Therefore, the development of devices for the detection of altered levels of IL-6 in low symptomatic people is desirable for prevention and early detection of insurgency of inflammation condition.

We have analyzed some IL-6 receptors, with the aim of revealing their possible performances when used as the active part of a IL-6 biosensor. We have compared some topological characteristics of the associate interaction networks. A quite interesting representation of these networks is given in terms of their contact maps, CoMas, which represent the closest nodes, for an assigned cut-off distance. By analyzing the CoMas, it is possible to observe that the smallest receptor, a SOMAmer, made of 32 nodes, is able to set up a network of bindings as wide as those of the other receptors, which instead, are made of about 400 nodes. Furthermore, the SOMAmer pattern of links is very similar to that produced by IL-6 with its primeval receptors, IL-6R and gp130. Specifically, it shows many links with helix C, bound to gp130 in the natural assembly, which are poorly present in the other receptors. The CoMas of the analyzed three Fabs (two from Camelid antibodies, $61 \mathrm{H} 7$ and $68 \mathrm{~F} 2$, and one from a therapeutic antibody, Olokizumab-OKZ) reveal some specific features: $61 \mathrm{H} 7$ mainly binds the longest IL-6 helices (A, B, C, D); OKZ has several links with the short helices E, F; finally, 68F2 binds helices $\mathrm{A}, \mathrm{D}$ and the loop between $\mathrm{A}$ and $\mathrm{B}$. These differences are due to the different orientations of the receptors toward IL-6, which, on the other side, reflect the different inhibition mechanisms activated by the receptors.

This analysis has been complemented with a percolation-like procedure which allows to analyze the surface complementarity of the receptor-ligand pair. To this aim, we have introduced an indicator, named ToCI (topological complementarity index) and ranked the receptors accordingly. Most of the analyzed receptors perform better than the natural assembly, and this result agrees with their high affinity to the cytokine. Finally, we have compared the expected resistance response of IL-6 when complexed with the analyzed receptors and have endorsed the use of Fab 61H7 and SOMAmer SL1025 for an electrochemical biosensor. The strategy that we have described is 
straightforward to implement and may be used to compare different receptors of a specific ligand, when their 3D structure is known, by crystallographic data or in silico procedures.

\section{Conclusions}

IL-6 cytokine early detection is an important tool for diagnosis and prevention of several diseases characterized by severe inflammatory states, from cancer to COVID-19. The use of Aptamers or Fabs as biosensing elements is often constrained by the specific skills of the researchers, and the general requirements of rapid implementation and cost effectiveness. The present theoretical investigation suggests that a new generation aptamer, SOMAmer SL1025, and a Camelid Fab, 61H7, are potentially very good candidates for the production of a IL-6 biosensor. Testing our prediction in a fully-fledged experimental setup seems worth exploring and could enlarge the available set of feasible IL-6 diagnostic tools.

Author Contributions: Conceptualization, E.A., M.B., M.C.; investigation; writing-original draft preparation, E.A.; writing-editing, E.A.; M.C.; writing-review and editing, E.A., M.B. All authors have read and agreed to the published version of the manuscript.

Funding: This research was supported in part by the Italian Ministry of Education, University and Research (MIUR) with the grant Project FFABR 2017.

Conflicts of Interest: The authors declare no conflict of interest.

\section{Appendix A}

In a realistic device, electrodes connect the biosensing element (receptors) to the electronic transducer. Therefore, the electrical response due to the binding process, has been simulated putting the electrodes on the receptor, specifically, on the first and last element. In doing so, we calculated the relative resistance of the complex vs the receptor, $r r=r_{\text {complex }} / r_{\text {receptor, }}$ Figure A1. We recall that the receptor is evaluated in its active state. In Figure A1 we report the spectra of relative resistance for the set of analyzed receptors, and, (green dashed line), the ratio of the resistance of the IL-6. IL-6R complex to the resistance of IL-6R in its native state (PDB ID: 1n26 [46]). As argued in Section 3.2, by increasing $D$, this ratio converges to the $r r$ of the IL-6. IL-6R complex (black line)

As discussed in Section 3.2, and reported in Figure A1, we focused our attention on the sensitive $D$ region, i.e. from the maximum of $r r$ to the the $90 \%$ of the difference between the maximum of $r r$ and its asymptotic value, here marked with a dot.

Notice that the analyzed receptors bind IL-6 in quite different way. A list of the 3D structures of the cytokine and the different complexes is given in Figure A2.

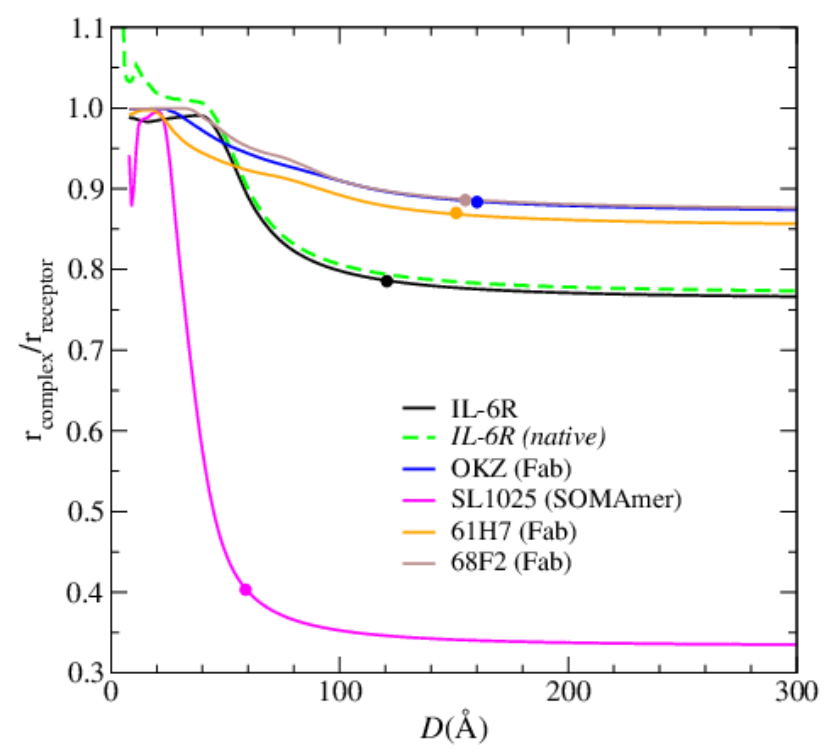


Figure A1. Relative resistance of IL-6 complexed with different receptors; contacts on the receptor. Dashed line describes the relative resistance of IL-6 complexed with IL-6R vs. IL-6R in its native state (PDB ID: 1 26 [46]).

$68 \mathrm{~F} 2$
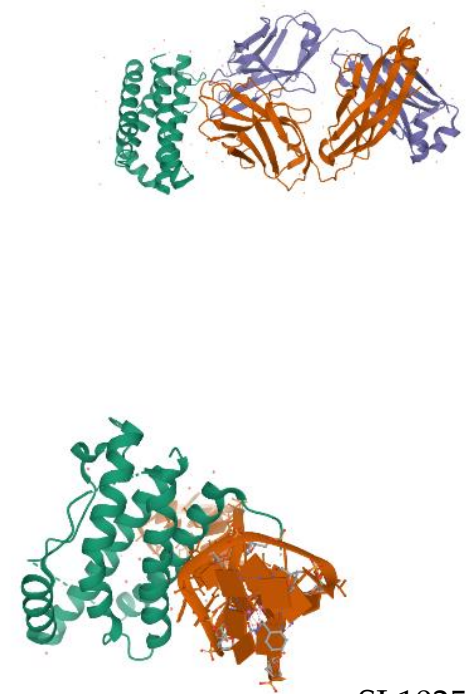

SL1025
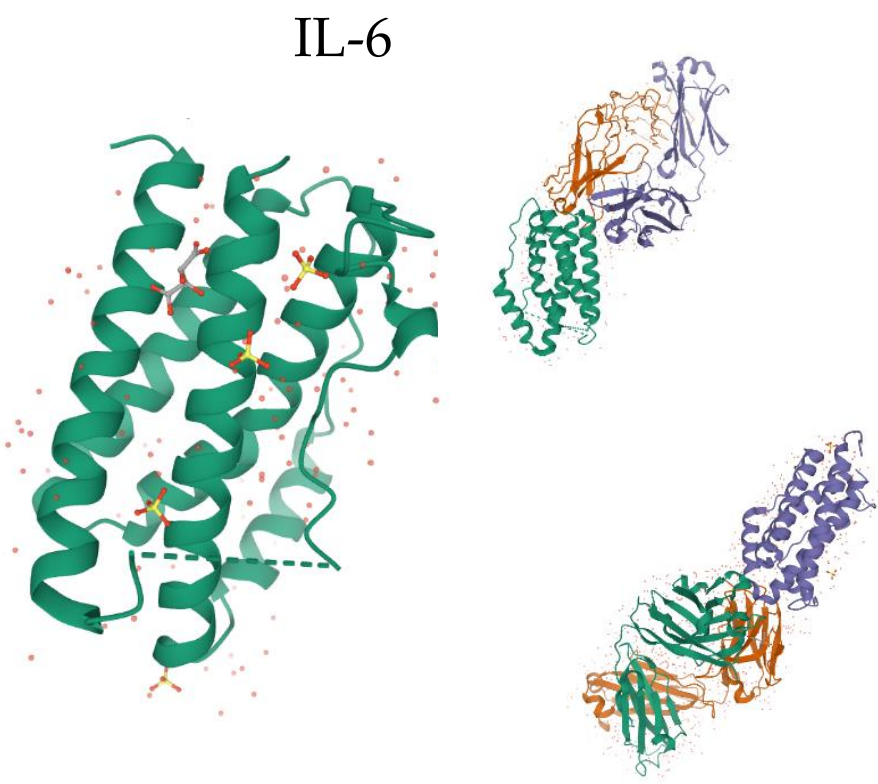

$\mathrm{OKZ}$
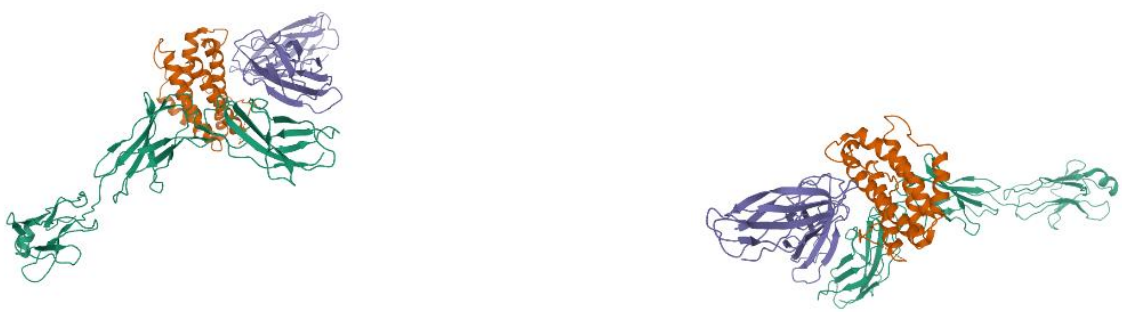

IL-6R \& gp130

Figure A2. The 3D pictures of IL-6 alone (in the middle) and conjugated with different receptors. The complex IL-6 gp130 - IL-6R is given in two different orientations (IL-6R in blue and green, gp130 in green). Font: The Protein Data Bank [38].

\section{References}

1. Mehta, P.; McAuley, D.F.; Brown, M.; Sanchez, E.; Tattersall, R.S.; Manson J.J. COVID-19: consider cytokine storm syndromes and immunosuppression. The Lancet 2020, 395, P1033-1034. doi: 10.1016/S0140-6736(20)30628-0

2. Moore, J. B.; June, C. H. Cytokine release syndrome in severe COVID-19. Science, 368(6490) 2020, 473-474. doi: $10.1126 /$ science.abb8925.

3. Hunter, C. A.; Jones, S. A. IL-6 as a keystone cytokine in health and disease. Nature immunology 2015, 16(5), 448-457.doi: https://doi.org/10.1038/ni.3153

4. Jones, S.A.; Scheller, J.; Rose-John, S. Therapeutic strategies for the clinical blockade of IL-6/gp130 signaling, J. Clin. Invest. 2011, 121 (9) 3375-3383. doi: 10.1172/JCI57158

5. Kontzias, A.; Kotlyar, A.; Laurence, A.; Changelian, P.; O'Shea J.J., Jakinibs: a new class of kinase inhibitors in cancer and autoimmune disease, Curr. Opin.Pharmacol. 2012, 12 (4), 464-470. doi: $\underline{10.1016 / j . c o p h .2012 .06 .008}$ 
6. Heinrich, P.C.; Castell, J.V.; Andus, T. Interleukin-6 and the acute phase response. Biochem J. 1990, 265 : 621-636.doi: https://doi.org/10.1042/bj2650621

7. Sato, K.; Tsuchiya, M.; Saldanha, J.; Koishihara, Y.; Ohsugi, Y.; Kishimoto, T.; Bendig, M.M. Reshaping a human antibody to inhibit the interleukin 6-dependent tumor cell growth. Cancer Res. 1993, 53: 851-856.

8. Sciascia, S.; Aprà F.; Baffa, A.; Baldovino, S.; Boero, D.; Bonora, S.; Calcagno, A.; Cecchi, I.; Cinnirella, G.; Converso, M.; Cozzi,M.; Crosasso P.; et al. Pilot prospective open, single-arm multicentre study on off-label use of tocilizumab in patients with severe COVID-19. Clin. Exp. Rheumatol. 2020, 38: 529-32.

9. Toniati, P.; Piva, S.; Cattalini, M.; Garrafa, E.;Regola, F.; Castelli,F.; Franceschini, F.; Airò P.; Bazzani, C.; Beindorf, E.-A.; Berlendi, M.; Bezzi, M.; et al. Tocilizumab for the treatment of severe COVID-19 pneumonia with hyperinflammatory syndrome and acute respiratory failure: a single center study of 100 patients in Brescia, Italy. Autoimmun. Rev. 2020, 19(7),102568. doi: 10.1016/j.autrev.2020.102568

10. Cavalli, G.; De Luca, G.; Campochiaro, C.; Della Torre, E.; Ripa, M.; Canetti, D.; Oltolini, C.; Castiglioni, B.; Tassan Din, C.; Boffini, N.; Tomelleri, A.; Farina, N. et al. Interleukin-1 blockade with high-dose anakinra in patients with COVID-19, acute respiratory distress syndrome, and hyperinflammation: a retrospective cohort study. Lancet Rheumatol. 2020, 2, e325-31, doi:https://doi.org/10.1016/S2665-9913(20)30127-2

11. Hosseini, F.; Hassannia, H.; Mahdian-Shakib, A.; Jadidi-Niaragh, F.; Enderami, S.E.; Fattahi, M.; Anissian,A.; Mirshafiey, A.; Kokhaei, P. Targeting of crosstalk between tumor and tumor microenvironment by $\beta$-D mannuronic acid (M2000) in murine breast cancer model. Cancer Med. 2017, 6 (3), 640-650. doi: $10.1002 / \mathrm{cam} 4.1013$

12. Taniguchi, K.; Karin, M. NF- $\mathrm{B}$, inflammation, immunity and cancer: coming of age. Nature Reviews Immunology 2018, 18(5), 309-324.doi: 10.1038/nri.2017.142

13. Bromberg, J.; Wang, T. C. Inflammation and cancer: IL-6 and STAT3 complete the link. Cancer cell 2009, 15(2), 79-80. doi:10.1016/j.ccr.2009.01.009

14. Grivennikov, S.; Karin, M. Autocrine IL-6 signaling: a key event in tumorigenesis?. Cancer cell 2008, 13(1), 7-9. doi: $\underline{10.1016 / j . c c r .2007 .12 .020}$

15. Ghandadi, M.; Sahebkar, A. Interleukin-6: a critical cytokine in Cancer multidrug resistance, Curr. Pharm. Des. 2016, 22 (5), 518-526. doi:10.2174/1381612822666151124234417

16. Kishimoto T. Interleukin-6: From basic science to medicine-40 years in immunology. Annu. Rev. Immunol. 2005, 23, 1-21. doi: 10.1146/annurev.immunol.23.021704.115806

17. Kumari, N.; Dwarakanath, B. S.; Das, A.; Bhatt, A. N. Role of interleukin-6 in cancer progression and therapeutic resistance. Tumor Biology 2016, 37(9), 11553-11572. doi:10.1007/s13277-016-5098-7

18. Shaw, S.; Bourne, T.; Meier, C.; Carrington, B.; Gelinas, R., Henry, A.; Popplewell, A.; Adams, R.; Baker, T.; Rapecki, S. Marshall, D.; Moore, A.; Neale, H.; Lawson, A. Discovery and characterization of olokizumab: a humanized antibody targeting interleukin-6 and neutralizing gp130-signaling. $m A b s$ 2014, 6, 773-781.doi: https://doi.org/10.4161/mabs.28612

19. Baraket, A.; Lee, M.; Zine, N.; Sigaud, M.; Bausells, J.; Errachid, A. A fully integrated electrochemical biosensor platform fabrication process for cytokines detection. Biosens. Bioelect. 2017, 93, 170-175.doi: https://doi.org/10.1016/j.bios.2016.09.023

20. Tertiş, M.; Ciui, B.; Suciu, M.; Săndulescu, R.; Cristea, C. Label-free electrochemical aptasensor based on gold and polypyrrole nanoparticles for interleukin 6 detection. Electrochim. Acta 2017, 258, 1208-1218.doi: https://doi.org/10.1016/j.electacta.2017.11.176

21. Tertis, M.; Leva, P. I.; Bogdan, D.; Suciu, M.; Graur, F.; Cristea, C. . Impedimetric aptasensor for the label-free and selective detection of Interleukin-6 for colorectal cancer screening. Biosens. Bioelectr. 2019, 137, 123-132.doi: https://doi.org/10.1016/j.bios.2019.05.012

22. Yang, T.; Wang, S.; Jin, H.; Bao, W.; Huang, S.; Wang, J. An electrochemical impedance sensor for the label-free ultrasensitive detection of interleukin-6 antigen. Sens. Act. B: Chemical 2013,178, 310-315.doi: https://doi.org/10.1016/j.snb.2012.12.107

23. Li, T.; Yang, M. Electrochemical sensor utilizing ferrocene loaded porous polyelectrolyte nanoparticles as label for the detection of protein biomarker IL-6. Sens. Act. B: Chemical 2011, 158(1), 361-365.doi: https://doi.org/10.1016/j.snb.2011.06.035

24. Huang, J.; Harvey, J.; Fam, W. D.; Nimmo, M. A.; Tok, I. A. Novel biosensor for Interleukin-6 detection. Procedia Engineering 2013, 60, 195-200. doi: 10.1016/j.proeng.2013.07.042 
25. Crivianu-Gaita, V.; Thompson, M. Aptamers, antibody scFv, and antibody Fab'fragments: An overview and comparison of three of the most versatile biosensor biorecognition elements. Biosens. Bioelectr. 2016, 85, 32-45.doi: https://doi.org/10.1016/j.bios.2016.04.091

26. Boulanger, M. J.; Chow, D. C.; Brevnova, E. E.; Garcia, K. C. Hexameric structure and assembly of the interleukin-6/IL-6 $\alpha$-receptor/gp130 complex. Science 2003, 300(5628), 2101-2104. doi: 10.1126/science.1083901

27. White, U.; M Stephens, J. The gp130 receptor cytokine family: regulators of adipocyte development and function. Curr. Pharm. Des. 2011, 17(4), 340-346. doi:https://doi.org/10.2174/138161211795164202

28. Klarenbeek, A.; Blanchetot, C.; Schragel, G.; Sadi, A. S.; Ongenae, N.; Hemrika, W.; Wijidenes, H.; Spinelli, S.; Desmyter, A.; Cambillau, C.; Hultberg, A.; Kretz-Rommel, A.; Dreier, T.; De Haard, H.J. W.; Roovers, R.C. Combining somatic mutations present in different in vivo affinity-matured antibodies isolated from immunized Lama glama yields ultra-potent antibody therapeutics. PEDS 2016, 29(4), 123-133.doi: https://doi.org/10.1093/protein/gzw003

29. Blanchetot, C.; De Jonge, N.; Desmyter, A.; Ongenae, N.; Hofman, E.; Klarenbeek, A.; Sadi, A.; Hultberg, A.; Spinelli, S.; Loris, R, Cambillau, C.; De Haard, H. Structural mimicry of receptor interaction by antagonistic interleukin-6 (IL-6) antibodies. J. Bio. Chem. 2016, 291(26), 13846-13854. doi: 10.1074/jbc.M115.695528

30. Lu, H., Tonge, P. J. Drug-target residence time: critical information for lead optimization. Current opinion in chemical biology 2010, 14(4), 467-474. https://doi.org/10.1016/j.cbpa.2010.06.176

31. Bonanni, B.; Kamruzzahan, A. S. M.; Bizzarri, A. R.; Rankl, C.; Gruber, H. J.; Hinterdorfer, P.; Cannistraro, S. Single molecule recognition between cytochrome C 551 and gold-immobilized azurin by force spectroscopy. Biophysical journal 2005, 89(4), 2783-2791.doi: https://doi.org/10.1529/biophysj.105.064097

32. Gelinas, A. D.; Davies, D. R.; Edwards, T. E.; Rohloff, J. C.; Carter, J. D.; Zhang, C.; Gupta S.; Ishikawa Y.;Hirota M.; Jarvis, T. C.; Neboisa J. Crystal structure of interleukin-6 in complex with a modified nucleic acid ligand. J.Bio. Chem. 2014, 289(12), 8720-8734.doi: 10.1074/jbc.M113.532697

33. Tuerk, C.; Gold, L. Systematic evolution of ligands by exponential enrichment: RNA ligands to bacteriophage T4 DNA polymerase. Science 1990, 249(4968), 505-510. doi: 10.1126/science.2200121

34. Jo, H.; Ban, C. Aptamer-nanoparticle complexes as powerful diagnostic and therapeutic tools. Experimental \& molecular medicine 2016, 48(5), e230-e230. doi: 10.1038/emm.2016.44

35. Tombelli, S.; Minunni, M.; Mascini, M. Aptamers-based assays for diagnostics, environmental and food analysis. Biomolecular engineering 2007, 24(2), 191-200. doi: https://doi.org/10.1016/j.bioeng.2007.03.003

36. Cataldo, R.; De Nunzio, G.; Millithaler, J. F.; Alfinito, E. Aptamers Which Target Proteins: What Proteotronics Suggests to Pharmaceutics. Current Pharmaceutical Design 2020, 26(3), 363-371.doi: $\underline{10.2174 / 1381612826666200114095027}$

37. Russo Krauss, I.; Merlino, A.; Randazzo, A.; Novellino, E.; Mazzarella, L.; Sica, F. (2012). High-resolution structures of two complexes between thrombin and thrombin-binding aptamer shed light on the role of cations in the aptamer inhibitory activity. Nucleic acids research 2012, 40(16), 8119-8128. doi: 10.1093/nar/gks512

38. Berman, H. M.; Westbrook, J.; Feng, Z.; Gilliland, G.; Bhat, T. N.; Weissig, H.; Shindyalov I.N.; Bourne, P. E. The protein data bank. Nucleic acids research 2000, 28(1), 235-242. doi: 10.1093/nar/gky1004.

39. Bini, A.; Mascini, M.; Mascini, M.;Turner, A. P. (2011). Selection of thrombin-binding aptamers by using computational approach for aptasensor application. Biosensors and Bioelectronics 2011, 26(11), 4411-4416. doi: $10.1016 /$ j.bios.2011.04.053

40. Cataldo, R.; Ciriaco, F.; Alfinito, E. A validation strategy for in silico generated aptamers. Computational biology and chemistry 2018, 77, 123-130.

41. Cataldo, R.; Giotta, L.; Guascito, M. R.; Alfinito, E. Assessing the quality of in silico produced biomolecules: the discovery of a new conformer. J. Phys. Chem. B 2019, 123(6), 1265-1273. https://doi.org/10.1021/acs.jpcb.8b11456

42. Alfinito, E.; Pousset, J.; Reggiani, L. Proteotronics: development of protein-based electronics. CRC Press. Boca Raton, FL 2015, pp1-270

43. Gold, L.; Ayers, D.; Bertino, J.; Bock, C.; Bock, A.; Brody, E., ... Zichi, D. Aptamer-based multiplexed proteomic technology for biomarker discovery. PLOS ONE 2010, 1, 1-77.doi: https://doi.org/10.1371/journal.pone.0015004 
44. Alfinito, E.; Reggiani, L. Opsin vs opsin: New materials for biotechnological applications. JAP 2014, 116(6), 064901.doi: https://doi.org/10.1063/1.4892445

45. Somers, W.; Stahl, M.; Seehra, J. S. 1.9 Å crystal structure of interleukin 6: implications for a novel mode of receptor dimerization and signaling. EMBO J. 1997 , 16(5), 989-997.doi: https://doi.org/10.1093/emboj/16.5.989

46. Varghese, J. N.; Moritz, R. L.; Lou, M. Z.; Van Donkelaar, A.; Ji, H.; Ivancic, N.; Branson, K.M.; Hall, N.E.; Simpson, R. J. Structure of the extracellular domains of the human interleukin-6 receptor $\alpha$-chain. PNAS 2002, 99(25), 15959-15964.doi: https://doi.org/10.1073/pnas.232432399

47. Kovacevic, K. D.; Gilbert, J. C.; Jilma, B. Pharmacokinetics, pharmacodynamics and safety of aptamers. Advanced drug delivery reviews 2018, 134, 36-50. doi: 10.1016/j.addr.2018.10.008

48. Gelinas, A. D., Davies, D. R., \& Janjic, N. Embracing proteins: structural themes in aptamer-protein complexes. Current opinion in structural biology 2016, 36, 122-132. doi: https://doi.org/10.1016/j.sbi.2016.01.009

49. Gupta, S.; Hirota, M.; Waugh, S. M.; Murakami, I.; Suzuki, T.; Muraguchi, M., Shibamori, M.; Ishikawa, Y.; Jarvis, C.T.; Carter, J.D.; Zhang, C.; Gawande, B.; Vrkljan, M.; Janjic, N.; Schneider, D.J. Chemically modified DNA aptamers bind interleukin-6 with high affinity and inhibit signaling by blocking its interaction with interleukin-6 receptor. J. Bio. Chem. 2014, 289(12), 8706-8719. doi: 10.1074/jbc.M113.532580

50. Nasonov E.; Fatenejad S.; Korneva E.; Krechikova D.; Maslyansky A.; Plaksina T.; Stanislav M.; Stoilov R.; Tyabut T.; Yakushin S.; Zonova E.; Genovese M. Safety and Efficacy of Olokizumab in a Phase III Trial of Patients with Moderately to Severely Active Rheumatoid Arthritis Inadequately Controlled by Methotrexate - CREDO1 Study. Arthritis Rheumatol. 2019; 71 (suppl 10). https://acrabstracts.org/abstract/safety-and-efficacy-of-olokizumab-in-a-phase-iii-trial-of-patients-with-m oderately-to-severely-active-rheumatoid-arthritis-inadequately-controlled-by-methotrexate-credo1-study/ . Accessed June 17, 2020.

51. Alfinito, E.; Reggiani, L.; Cataldo, R.; De Nunzio, G.; Giotta, L.; Guascito, M. R. Modeling the microscopic electrical properties of thrombin binding aptamer (TBA) for label-free biosensors. Nanotechnology 2017, 28(6), 065502. doi: 10.1088/1361-6528/aa510f

52. Bizzarri, A. R., Cannistraro, S. The application of atomic force spectroscopy to the study of biological complexes undergoing a biorecognition process. Chemical Society Reviews 2010, 39(2), 734-749. doi: $10.1039 / \mathrm{b} 811426 \mathrm{a}$

53. Alfinito, E.; Reggiani, L. Mechanisms responsible for the photocurrent in bacteriorhodopsin. Phys. Rev. E 2015, 91(3), 032702. doi: 10.1103/PhysRevE.91.032702

54. Alfinito, E.; Millithaler, J. F.; Reggiani, L.; Zine, N.; Jaffrezic-Renault, N. Human olfactory receptor 17-40 as an active part of a nanobiosensor: a microscopic investigation of its electrical properties. Rsc Advances 2011, 1(1), 123-127. doi: $10.1039 /$ C1RA00025I

55. Kobilka, B. K.; Deupi, X. Conformational complexity of G-protein-coupled receptors. Trends in pharmacological sciences 2007, 28(8), 397-406. doi: 10.1016/j.tips.2007.06.003

56. Bochet, L.; Meulle, A.; Imbert, S.; Salles, B.; Valet, P.; Muller, C. Cancer-associated adipocytes promotes breast tumor radioresistance, Biochem. Biophys. Res. Commun. 2011, 411 (1), 102-106.doi: 10.1016/j.bbrc.2011.06.101

57. Bromberg,J.; Wang, T.C. Inflammation and cancer: IL-6 and STAT3 complete the link. Cancer Cell 2009, 15 (2), 79-80. doi: 10.1016/j.ccr.2009.01.009

58. Hirano, T.; Ishihara, K.; Hibi, M. Roles of STAT3 in mediating the cell growth, differentiation and survival signals relayed through the IL-6 family of cytokine receptors. Oncogene 200019 (21), 2548-2556. doi: 10.1038/sj.onc.1203551

59. Guo, Y.; Xu, F.; Lu, T.; Duan, Z.; Zhang, Z. Interleukin-6 signaling pathway in targeted therapy for cancer. Cancer Treat. Rev. 2012, 38 (7), 904-910.doi: https://doi.org/10.1016/j.ctrv.2012.04.007

60. Wolf, J.; Rose-John, S.; Garbers, C. Interleukin-6 and its receptors: a highly regulated and dynamic system, Cytokine 2014, 70 (1) 11-20.doi: 10.1016/j.cyto.2014.05.024 